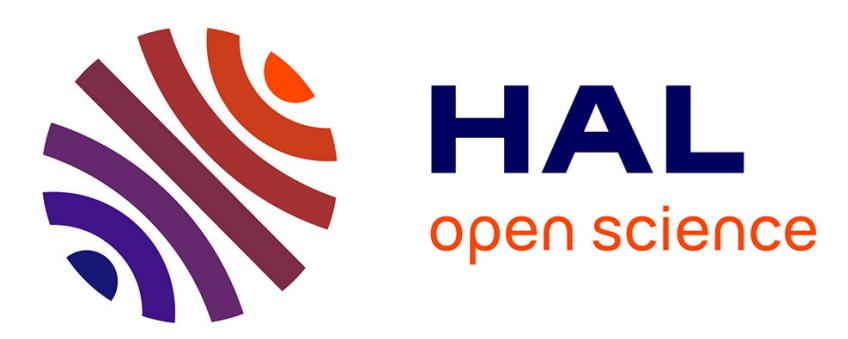

\title{
Strong turbulence for vibrating plates: Emergence of a Kolmogorov spectrum
}

\author{
Gustavo Düring, Christophe Josserand, Giorgio Krstulovic, Sergio Rica
}

\section{To cite this version:}

Gustavo Düring, Christophe Josserand, Giorgio Krstulovic, Sergio Rica. Strong turbulence for vibrating plates: Emergence of a Kolmogorov spectrum. Physical Review Fluids, 2019, 4 (6), 10.1103/PhysRevFluids.4.064804. hal-02172291

\section{HAL Id: hal-02172291 \\ https://hal.science/hal-02172291}

Submitted on 3 Jul 2019

HAL is a multi-disciplinary open access archive for the deposit and dissemination of scientific research documents, whether they are published or not. The documents may come from teaching and research institutions in France or abroad, or from public or private research centers.
L'archive ouverte pluridisciplinaire HAL, est destinée au dépôt et à la diffusion de documents scientifiques de niveau recherche, publiés ou non, émanant des établissements d'enseignement et de recherche français ou étrangers, des laboratoires publics ou privés. 


\title{
Strong turbulence for vibrating plates: Emergence of a Kolmogorov spectrum
}

\author{
Gustavo Düring, ${ }^{1}$ Christophe Josserand, ${ }^{2}$ Giorgio Krstulovic, ${ }^{3}$ and Sergio Rica ${ }^{2,4, *}$ \\ ${ }^{1}$ Instituto de Física, Pontificia Universidad Católica de Chile, Casilla 306, Santiago, Chile \\ ${ }^{2}$ LadHyX, CNRS and Ecole Polytechnique, UMR 7646, IP Paris, 91128 Palaiseau, France \\ ${ }^{3}$ Université Côte d'Azur, Observatoire de la Côte d'Azur, CNRS, Laboratoire Lagrange, \\ Boulevard de l'Observatoire, CS 34229, 06304 Nice cedex 4, France \\ ${ }^{4}$ Facultad de Ingeniería y Ciencias and UAI Physics Center, Universidad Adolfo Ibáñez, \\ Avenida Diagonal las Torres 2640, Peñalolén, Santiago, Chile
}

(Received 29 October 2018; published 13 June 2019)

\begin{abstract}
In fluid turbulence, energy is transferred from one scale to another by an energy cascade that depends only on the energy-dissipation rate. It leads by dimensional arguments to the Kolmogorov 1941 (K41) spectrum. Here we show that the normal modes of vibrations in elastic plates also manifest an energy cascade with the same K41 spectrum in the fully nonlinear regime. In particular, we observe different patterns in the elastic deformations such as folds, developable cones, and even more complex stretching structures, in analogy with spots, swirls, vortices, and other structures in hydrodynamic turbulence. We show that the energy cascade is dominated by the kinetic contribution and that the stretching energy is at thermodynamical equilibrium. We characterize this energy cascade, the validity of the constant energy-dissipation rate over the scales. Finally, we discuss the role of intermittency using the correlation functions that exhibit anomalous exponents.
\end{abstract}

DOI: 10.1103/PhysRevFluids.4.064804

\section{INTRODUCTION}

Turbulence has remained a central problem in fluid dynamics since the early experiments of Reynolds [1]. Perhaps the most salient feature is an energy cascade that redistributes the energy among different Fourier modes of the velocity fluctuations that are independent of the dissipation caused by viscosity at small scales. This energy cascade depends only on the energy-dissipation rate per unit mass, $P$, and the wave number $k$ leading to the Kolmogorov K41 [2] spectrum:

$$
E_{k} \sim P^{2 / 3} k^{-5 / 3} \text {. }
$$

Despite a century of effort, turbulence still remains a major challenge from the experimental, theoretical, and numerical points of view, and the very essence of the phenomenon has not yet been revealed satisfactorily [3-5]. For instance, if the K41 law can be deduced using dimensional arguments, the statistical description of the turbulence fails to correctly describe the general correlation functions of the velocity fields. In fact, to date, there is only a single analytical result that may be derived from the original Navier-Stokes (NS) equations for incompressible fluids, the so-called von Kármán-Howarth relation [6], that links the second- and third-order correlation functions of some component of the fluid velocity with the energy-dissipation rate. Higher moments of these quantities differ from mean-field-based predictions. Experimental and numerical data reveal extreme events, exhibiting heavy-tailed distributions, that are usually associated with intermittency. Moreover, one can argue that the difficulty of describing turbulence may come from the fundamental

\footnotetext{
*sergio.rica@uai.cl
} 
issues raised by the fluid mechanics equations, witnessed in particular by the Clay millennium problem on the regularity of the NS equations in three space dimensions. Similarly, fluid turbulence is a hard problem because it is on the same footing as the Euler equation for perfect fluids which is ab initio a nonlinear partial-differential equation without any small parameter that may justify an asymptotic scheme nor a rational closure for the statistical correlations [7]. Since fluid turbulence is studied in the framework of the NS equations, it is important to question whether such cascade dynamics transporting the energy from the large injection scales to the small dissipative ones can be observed in different contexts and whether the turbulence features described in fluids are universal or dependent on the dynamical equations. In particular, while the quadratic nonlinear term of the NS equation is responsible for the mixing between scales at the heart of the cascade process, nothing in the theoretical description of turbulence takes into account the details of the nonlinearity itself. Fluidlike turbulence has also been observed in different contexts such as magnetohydrodynamics where the magnetic field is coupled with the flow [8] or viscoelastic turbulence in polymer solutions at low Reynolds numbers [9]. In these cases, even though the nonlinearity may differ from the one of NS equations and different spectra can be measured, they have similar properties as the original Kolmogorov phenomenology.

In this paper, we propose to investigate a turbulent regime in a totally different physical system: the vibrations of elastic plates in the full nonlinear limit. The dynamics is deduced from the Föpplvon Kármán equations [10,11] (described below) in the limit of vanishing plate thickness. Here, the nonlinearity of the equation comes from the geometry and is cubic, in contrast to the usual quadratic advection nonlinearity in fluids. However, such system has recently revealed a strong analogy with hydrodynamic turbulence through the derivation of an exact law equivalent to the Kolmogorov's 4/5 law [12]. For finite plate thickness, the small-scale dynamics are always dominated by elastic waves, and wave-turbulence regimes have been predicted theoretically, as well as observed numerically [13] and experimentally $[14,15]$. Such wave turbulence can be described using the weak or wave-turbulence theory (WTT), developed originally in the 1960s [16-26] and applied afterwards in many physical contexts such as surface gravity, capillary waves, plasmas [20-23], nonlinear optics [27], and, recently, gravitational waves in general relativity [28]. In the present work, the wave dynamics vanishes but a turbulent, fully nonlinear regime is still expected to exist and will be studied here precisely. For instance, this system will allow us to question the robustness of the statistical properties of a turbulent field when the nonlinearity varies. Such results should therefore reveal the role and the influence of the different terms of the NS equations in fluid turbulence.

\section{THEORETICAL MODEL: THE ZERO-THICKNESS FÖPPL-VON KÁRMÁN EQUATIONS}

The vibration of a bending-free elastic plate comes from the usual dynamical version of the Föppl-von Kármán equations [29] for the vertical amplitude of the deformation $\zeta(x, y, t)$ and for the Airy stress function $\chi(x, y, t)$ that describes the three in-plane stresses:

$$
\begin{gathered}
\rho \frac{\partial^{2} \zeta}{\partial t^{2}}=\zeta_{x x} \chi_{y y}+\zeta_{y y} \chi_{x x}-2 \zeta_{x y} \chi_{x y}+\mathcal{F}+\mathcal{D}, \\
\frac{1}{E} \Delta^{2} \chi=-\left(\zeta_{x x} \zeta_{y y}-\zeta_{x y}^{2}\right) .
\end{gathered}
$$

Here, $\Delta=\partial_{x x}+\partial_{y y}$ is the usual Laplacian and $\rho$ and $E$ are, respectively, the mass density and the Young modulus $E$ of the plate. $\mathcal{F}$ and $\mathcal{D}$ are the forcing and dissipation, respectively.

This derivation assumes that the in-plane displacements are negligible and a quasistatic force balance holds. This is taken into account through the Airy stress function $\chi(x, y, t)$ which follows the dynamics through Eq. (2). Finally, although the Föppl-von Kármán equation are valid for large deformations, the local curvature must be smaller than the inverse of the thickness of the plate.

When $\mathcal{F}=\mathcal{D}=0$, Eqs. (1) and (2) derive from a Hamiltonian principle [13,30]. More importantly, it is a well-posed system, in the sense that the energy $\mathcal{E}=\mathcal{E}_{\text {kin }}+\mathcal{E}_{\text {stret }}$ is composed of two 
positive (hence bound from below) quantities, namely, the kinetic energy and the stretching energy per unit mass, yielding, respectively,

$$
\begin{gathered}
\mathcal{E}_{\text {kin }}=\frac{1}{2 S} \int \dot{\zeta}^{2} d \boldsymbol{r}, \\
\mathcal{E}_{\text {stret }}=\frac{E}{8 \rho S} \int\left[\Delta^{-1}\left(\zeta_{x x} \zeta_{y y}-\zeta_{x y}^{2}\right)\right]^{2} d \boldsymbol{r},
\end{gathered}
$$

where $S$ is the area of the plate. For dimensional purposes, we use everywhere the energies per unit mass, $\mathcal{E}, \mathcal{E}_{\text {kin }}$, and $\mathcal{E}_{\text {stret }}$, with dimensions of the square of a speed.

Contrary to fluid turbulence, where the energy, $\frac{1}{2} \int \boldsymbol{v}^{2} d \boldsymbol{r}$, comes from a single quadratic contribution, the energy in our system is composed of two contributions. In what follows, we will quantify the role of each contribution separately, but keep in mind that only the total energy is conserved. Finally, it is important to notice that the nonlinearity of Eq. (1) is cubic since the Airy function depends quadratically on the deformation field $\zeta$. Our system of equations can thus be considered as the cubic counterpart of the NS equation and it will be interesting to investigate if and how it can exhibit turbulent behavior.

\section{TURBULENT BEHAVIOR}

We performed numerical simulations of Eqs. (1) and (2) by using a standard pseudospectral method in a periodic domain with an additive random forcing at large scales and a viscous damping acting at small scales. The explicit form of forcing and dissipation is discussed below. Turbulent states should not depend explicitly on these details, provided that these mechanisms are well separated in wave-number space.

We solve the Föppl-von Kármán equations (1) and (2) with a standard pseudospectral code in a square domain of size $2 \pi$ with periodic boundary conditions. The forcing $\mathcal{F}$ is white noise in time of variance $f_{0}^{2}$ and its Fourier modes are nonzero only for wave vectors $1.5 \leqslant|k| \leqslant 3$. De-aliasing is made by using the standard $2 / 3$ rule that is applied after computing each quadratic term. The dissipation is acting at small and large scales in the following way:

$$
\mathcal{D}=-v(-\Delta)^{n_{v}} \dot{\zeta}-\alpha(-\Delta)^{-n_{\alpha}} \dot{\zeta}
$$

Figures 1(a) and 1(b) show the surface plate deformation $\zeta(x, y)$ and the plate vertical speed $\dot{\zeta}(x, y)$, respectively.

The displacement field displays a coarse scale, superimposed with fine scale fluctuations composed of a large number of wrinkles of various sizes. Such multiscale states are the most prominent characteristic of turbulent systems. The local speed of the plate in Fig. 1(b) demonstrates that the elastic plate is not at equilibrium, as it displays a myriad of excited modes. When bending is absent, it is natural to expect the appearance of highly nonlinear geometrical structures. Such structures are visible in Fig. 1(c), which shows a closeup of the plate deflection. The small-scale wrinkles consist of a random assembly of moving ridges and conical points. Ridges and conical points are, in fact, the fundamental equilibrium configurations of elastic plates in the bending-free limit. These structures have been extensively studied since the pioneering works performed in the 1990s [31-33]. In the bending-free limit, the elastic deformations favor the bending modes because they cost no energy. However, because of geometrical constraints, it is not always possible to have a fully developable surface everywhere and the system thus creates singular structures: linear ridges [31] and pointy developable cones or, more commonly named, D cones [32]. The deformations of the plates are then controlled by the stretching that modifies the locally plane metric of the sheet since these geometrical structures concentrate the plate curvature and the stretching energy. If the plate thickness is small but finite, these singular structures are eventually regularized by the bending. Here, in the absence of bending, the viscous term appears to be the only possible mechanism for regularization. The dynamics of a bending-free elastic plate thus corresponds to a 

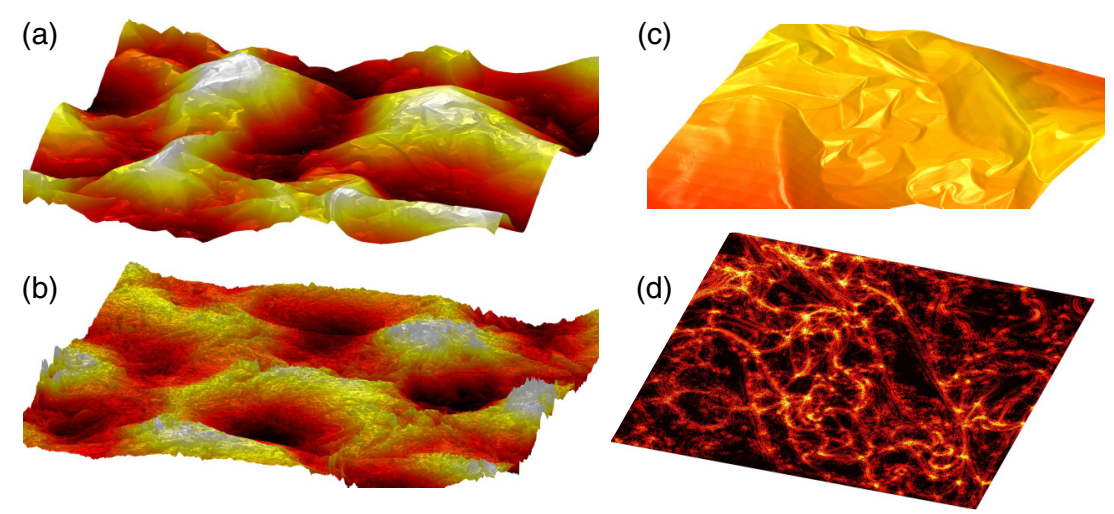

FIG. 1. Turbulent states of a bending-free plate. (a) Surface plot of the plate deformation $\zeta(x, y)$. (b) Surface plot of the plate vertical velocity $\dot{\zeta}(x, y)$. (c) Detail of the wrinkles in a zoom of the surface plot of the plate deformation $\zeta(x, y)$. (d) Snapshot of the $G(x, y)(6)$ of the respective area. The density plot displays the values of $\ln _{10}|G|$. Simulations were made at resolution of $4096^{2}$, and the parameters values are $n_{v}=3$, $v=2 \times 10^{-12}, n_{\alpha}=2, \alpha=100$, and $f_{0}=1$.

myriad of singularities regularized by the dissipation and moving (apparently) randomly over the sheet. See Supplemental Material [34] for a movie of these defect-induced dynamics. It shows the plate deformation $\zeta(x, y)$ surface plot for turbulent states of a bending-free plate. Simulations were made at resolution of $4096^{2}$ with an additive random forcing.

To precisely catch these singular structures of dimension 1 (ridges) and 2 (D cones), we have plotted in Fig. 1(d) the Hessian written in the right-hand side of Eq. (2), which is also the dominant order correction to the instantaneous Gaussian curvature,

$$
G(x, y, t)=\zeta_{x x} \zeta_{y y}-\zeta_{x y}^{2}
$$

which, after (2), is the source of the in-plane stresses via the Airy function, $\chi(x, y, t)$. This quantity exhibits the expected complex network of ridges and D cones.

An immediate analogy can be established with hydrodynamic turbulence. Fully nonlinear elastic plate turbulence seems to be characterized by a "crumpling cascade," where wrinkles play the role of whirls and, instead of vortex filaments and sheets, the localized singularities come in the form of ridges and D cones. To illustrate this idea further, we integrate the system (1) and (2) starting from a flat state, with no forcing $(\mathcal{F}=0)$, but with an initial velocity at large scales, and let the system decay. Snapshots of the velocity field, shown in Fig. 2, exhibit the dynamics of this decaying turbulence configuration.

Initially, all the velocity fluctuations are contained at large scales; see Fig. 2(a). Then, due to the nonlinear interactions, instabilities appear creating smaller and smaller structures [see Fig. 2(b)]. After this transient, a turbulent state is observed where fluctuations at all scales coexist [Fig. 2(c)]. Finally, at large times, the dissipative terms acting at small scales kill the turbulent state, smoothing out the fluctuations [see Fig. 2(d)].

\section{A. Kolmogorov spectrum}

By forcing at large scales and dissipating at small scales, a turbulent out-of-equilibrium steady state is obtained, such as the one observed in Fig. 1. A quantitative measurement of these steady turbulent states is obtained through the spectral densities of the plate velocity. As in fluid turbulence, here we compute the average kinetic-energy spectrum and the kinetic-energy flux for different wave numbers. The kinetic-energy spectrum per unit mass, $E_{\text {kin }}(k)$, is defined through the kinetic energy 

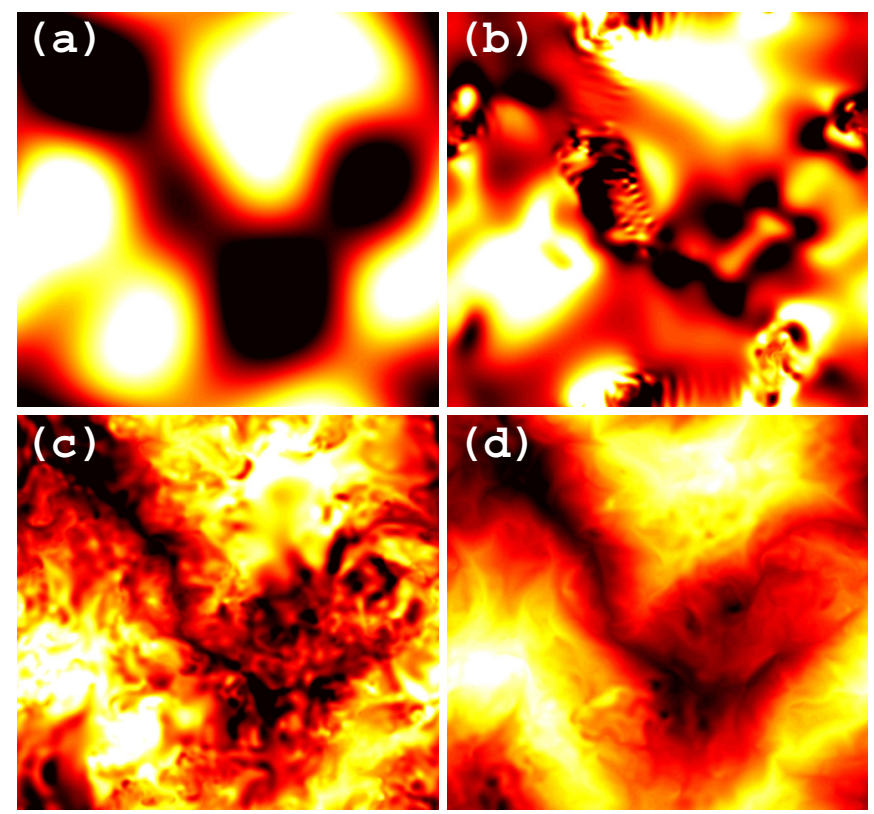

FIG. 2. Simulations for decaying turbulence. Snapshots of $\dot{\zeta}$. Time goes as (a) $t=0$, (b) $t=0.5$, (c) $t=$ 65 , and (d) $t=100$ [in time units of (1) and (2)]. The simulations were performed with $1024^{2}$ collocation points, $\mathcal{F}=0, n_{v}=2, v=4 \times 10^{-6}, \alpha=0$, and a random initial condition at large scale only on $\dot{\zeta}$.

per unit mass by

$$
\mathcal{E}_{\text {kin }}=\int E_{\text {kin }}(k) d k,
$$

where $E_{\text {kin }}(k)=2 \pi k\left\langle\left|\dot{\zeta}_{k}\right|^{2}\right\rangle$, the velocity-field Fourier transform is $\dot{\zeta}_{k}(t)=\int \dot{\zeta}(\boldsymbol{r}, t) e^{i \boldsymbol{k} \cdot \boldsymbol{r}} d \boldsymbol{r}$, and isotropy is assumed. In addition, the kinetic-energy flux $P_{\text {kin }}(k)$ is defined through the transfer equation,

$$
\frac{\partial}{\partial t} E_{\text {kin }}(k)=-\frac{\partial}{\partial k} P_{\text {kin }}(k) .
$$

Since $E_{\text {kin }}(k)$ has dimensions of $L^{3} / T^{2}$, the dimensions of $P_{\text {kin }}(k)$ are $L^{2} / T^{3}$. Notice that the stretching energy (4) is also a quadratic quantity, and analogous definitions can be given for its spectrum and flux (see Appendix A for explicit definitions).

We have directly measured the energy fluxes for various turbulent runs with different forcing amplitudes. Figure 3(a) shows the time-averaged kinetic-energy (solid lines) fluxes, normalized by their mean value $\bar{P}_{\text {kin }}$ in the well-defined transparency window where they are flat.

This transparency window is usually called the inertial range in the context of hydrodynamic turbulence. At small scales, dissipation takes place and produces, at high forcing, a bottleneck that invades the inertial range as the mean energy flux increases, although an inertial range is still clearly present. Such a bottleneck effect has already been observed in hydrodynamic turbulence $[7,35]$ and is usually attributed to an insufficient inertial range. We suggest that in our numerics, the observed bottleneck is probably a consequence of the hyperviscosity and the strong energy transfer inhibition that leads to partial thermalization. Figure 3(a) also displays the stretching energy fluxes (dashed lines), which are notably smaller than the kinetic-energy fluxes. Therefore, numerical simulations indicate that the pertinent energy flux for the cascade is the kinetic-energy one because it shows a constant energy flux along scales in an inertial range. A constant energy flux is usually associated with a turbulent regime that leads to a power-law behavior for the corresponding energy spectrum. 

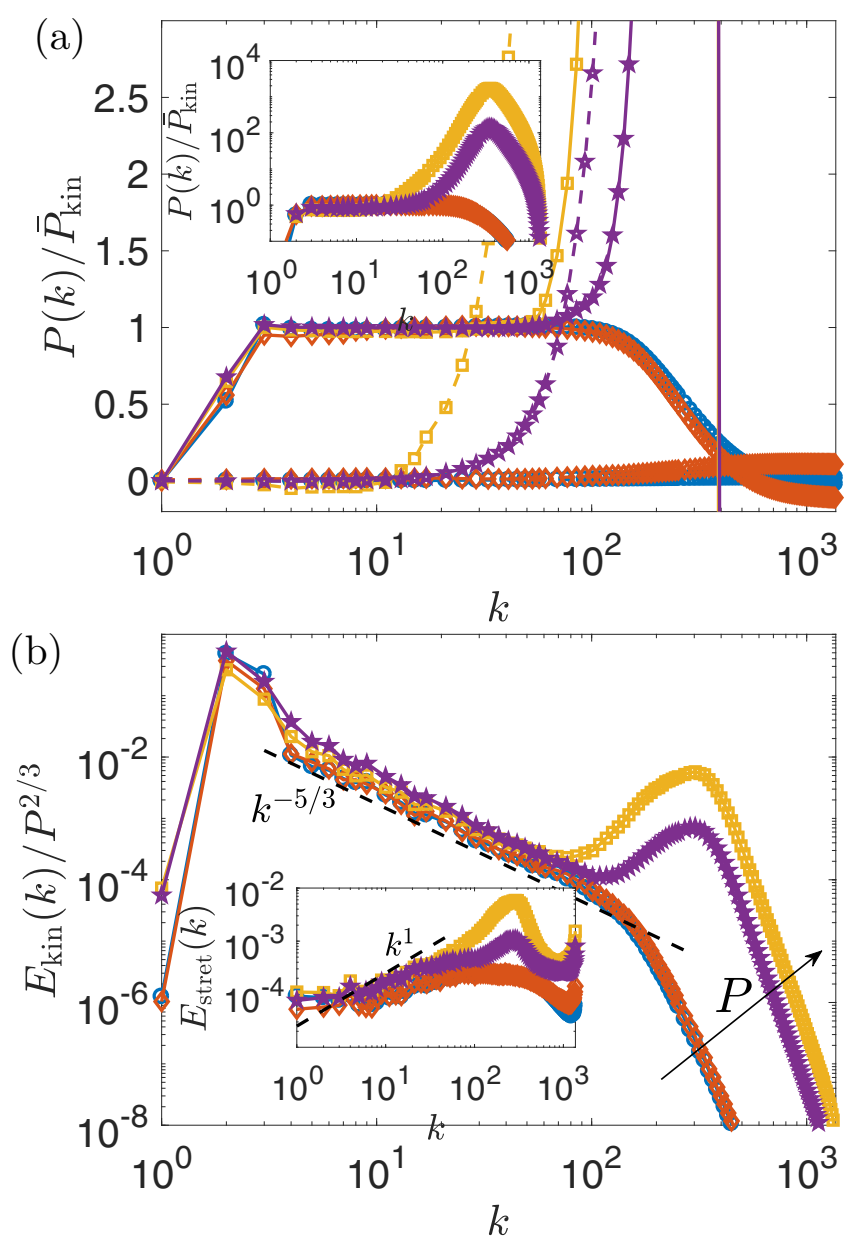

FIG. 3. (a) Time-averaged kinetic-energy flux (solid lines) and the stretching energy flux (dashed lines). Different markers (and colors) are for different runs with increasing forcing amplitude or fluxes [see arrow in (b)]. The fluxes have been normalized by their total mean flux value in the inertial range (see methods for values). The inset displays the same plot but in log-log. (b) Time-averaged kinetic-energy spectra normalized by $P^{2 / 3}$. The dashed line displays the Kolmogorov scaling. Inset: Time-averaged stretching energy. The dashed line displays the thermalization energy scaling. The arrow indicates the different runs with increasing values of the flux $P$. The simulations were performed with the same parameters of Fig. 1 for different forcing amplitudes, $f_{0}=1,8,27$, and 100 , and the measured values of the fluxes were $\bar{P}_{\text {kin }}=\{0.0284,0.0354,5.3358,16.5325\}$, respectively.

This is indeed the case for the kinetic-energy spectrum, as shown in Fig. 3(b), where the $k^{-5 / 3}$ Kolmogorov law is apparent.

The kinetic turbulent spectrum can be discussed on dimensional grounds in the footsteps of Kolmogorov. However, here in addition to the constant energy flux per unit mass $P$ and the wave number $k$ (with dimension inverse of a length), the spectrum should depend a priori on the additional material parameter $E / \rho$. Since a characteristic length can be defined, namely, $\lambda=(E / \rho)^{3 / 2} / P$, one generically gets

$$
E_{\mathrm{kin}}(k)=\frac{P^{2 / 3}}{k^{5 / 3}} \Phi\left[k \frac{(E / \rho)^{3 / 2}}{P}\right],
$$


where $\Phi(\cdot)$ is an arbitrary function of the dimensionless argument $k \lambda$. Contrary to fluid turbulence, the existence of this extra length $\lambda$ does not allow us to uniquely determine a fixed exponent for the power-law spectrum. Remarkably, numerics suggest that the kinetic-energy and the stretching energy spectra can be treated independently. Considering that for the kinetic energy the elastic property of the material does not intervene, the kinetic-energy spectrum should follow

$$
E_{\text {kin }}(k)=C_{\text {kin }} P^{2 / 3} k^{-5 / 3},
$$

with $C_{\text {kin }}=\Phi(0) \neq 0$ a constant. The numerical simulations indeed show good agreement with this predicted scaling (9) for both $k$ and $P$, with $\lambda k$ spanning over four orders of magnitude $(0.24<$ $\left.\lambda k<2.8 \times 10^{3}\right)$.

\section{B. Stretching energy flux}

Because the total energy combines two different contributions (3) and (4), we address the role of the stretching energy (4). In the following, we show, via a simple argument, that the stretching energy flux related to the stretching energy must vanish in a statistically steady state under an additive forcing and a viscouslike dissipation, as the one considered in this work.

Following [12], we introduce the spatial correlation function

$$
\mathcal{E}_{\text {stret }}(\ell)=\frac{1}{2 E}\left\langle\Delta_{r} \chi(\boldsymbol{r}) \Delta_{r^{\prime}} \chi\left(\boldsymbol{r}^{\prime}\right)\right\rangle,
$$

with $\ell=\boldsymbol{r}^{\prime}-\boldsymbol{r}$, which in the limit of $\ell \rightarrow 0$ converges to the mean value of the stretching energy. Considering a homogeneous and isotropic system, it can then be shown that the time derivative of the correlation function (10) follows

$$
\begin{aligned}
\dot{\mathcal{E}}_{\text {stret }}(\ell) & =-\left\langle\chi\left(\boldsymbol{r}^{\prime}\right)\{\zeta(\boldsymbol{r}), \dot{\zeta}(\boldsymbol{r})\}\right\rangle \\
& =\frac{1}{V} \int \chi(\boldsymbol{r}+\boldsymbol{\ell})\{\zeta(\boldsymbol{r}), \dot{\zeta}(\boldsymbol{r})\} d \boldsymbol{r} .
\end{aligned}
$$

The last equality considers that for a homogeneous system, the statistical average can be taken as a spatial average. From the convolution theorem, we have that the Fourier transform $\dot{\mathcal{E}}_{\text {stret }}(\boldsymbol{p})=$ $\chi_{\boldsymbol{p}}\{\zeta, \dot{\zeta}\}_{-\boldsymbol{p}}$. In a steady state, the time derivative of the correlation function $\dot{\mathcal{E}}_{\text {stret }}(\boldsymbol{p})$ must be zero, and hence also $\chi_{p}\{\zeta, \dot{\zeta}\}_{-p}=0$. By definition, the stretching energy flux (see Appendix A) is

$$
P_{\text {stret }}(k)=\int_{0}^{k}\left\langle\chi_{\boldsymbol{p}}\{\zeta, \dot{\zeta}\}_{-p}\right\rangle d p
$$

and therefore $P_{\text {stret }}(k)$ must be zero.

This result follows directly from the observation that the stretching energy flux appears to be the time derivative of the stress correlation function, which must be zero in a statistically steady state. This is indeed the case, as is apparent in Fig. 3(a). Therefore, one would expect the stretching modes to eventually thermalize. Since the stretching energy (4) is quadratic in $\gamma(\boldsymbol{r})=\Delta^{-1}\left(\zeta_{x x} \zeta_{y y}-\zeta_{x y}^{2}\right)$, the equilibrium distribution corresponds to equipartition of the Fourier modes of $\gamma$. In particular, it leads to the equipartition spectrum of the form

$$
E_{\text {stret }}(k)=C_{\text {stret }} k,
$$

with $C_{\text {stret }}$ a constant proportional to the mean energy, which plays the role of an effective temperature. In the inset of Fig. 3, the stretching spectra for different forcing amplitude are shown to be consistent with the equipartition law (12), for the scales where the dissipation is negligible.

\section{Intermittency and beyond Kolmogorov phenomenology}

The Kolmogorov phenomenology discussed in the previous section is based on dimensional analysis and mean-field assumptions that neglect the existence of extreme fluctuations. Nevertheless, it is 
well known that deviations exist to such predictions and they become very important when looking at higher-order statistics [7]. These deviations are associated with the intermittent statistics of the fields and are somehow inherent to fluid turbulence, although a complete understanding of such fluctuations is still missing. Unlike hydrodynamic turbulence, the theory of weak wave turbulence predicts Gaussian statistics for the distribution of wave amplitudes; therefore no intermittency can be observed within the range of validity of the theory. However, when the nonlinearities become of the same order of the linear dispersive terms, the wave-turbulence theory breaks down and can manifest intermittent statistics [36]. Such intermittencies have been experimentally observed in gravity-capillary waves [37] and suggested for thin elastic plates [38]. Moreover, numerical simulations of elastic plates at strong forcing have shown important deviations from the WTT predicted scalings for the plate deformation [39,40], leading to intermittency signatures $[38,40]$.

Intermittency is usually addressed looking at the moments of the so-called structure functions of the fields, which provide information on the variation of the fields at a given scale [7]. For elastic plates, because of the fast decay of the deformation spectrum, a second-order difference is needed to observe an intermittent scaling [40]. In our system, where bending waves are absent and the dynamics is thus fully nonlinear, the intermittent behavior is expected to occur at any forcing. Following [40], we introduce the (second variation) increments $\delta_{\ell}^{2} \zeta$ of the plate deformation and their corresponding structure functions $S_{p}(\ell)$, namely,

$$
S_{p}(\ell)=\left\langle\left|\delta_{\ell}^{2} \zeta\right|^{p}\right\rangle
$$

with

$$
\delta_{\ell}^{2} \zeta=\zeta(x+\ell)-2 \zeta(x)+\zeta(x-\ell) .
$$

At very small scale, the regularity of $\zeta(x)$ implies the scaling $S_{p}(\ell) \sim \ell^{2 p}$. However, in the inertial range, a nontrivial scaling appears. We define then the anomalous exponents (in the spirit of hydrodynamic turbulence) $\xi_{p}$, from the moments following $S_{p}(\ell) \sim \ell^{\xi_{p}}$. From our numerics, we measure that $\xi_{2} \approx 2.2$. In Kolmogorov theory as well as for WTT, a linear relation between the different exponents is expected, $\xi_{p}=\frac{\xi_{2}}{2} p$, witnessing the self-similar nature of the dynamics. For vibrating elastic plates, however, a deviation from this linear law has been observed, indicating the presence of intermittency that breaks the self-similarity of crumpling dynamics [40]. In the bottom inset of Fig. 4, we present the local slope of the structure functions defined as $\xi_{p}(\ell)=\frac{d \ln S_{p}(\ell)}{d \ln \ell}$ for different orders $p$. A relatively flat behavior is observed in the inertial range and the anomalous exponents $\xi_{p}$ are measured by averaging $\xi_{p}(\ell)$ in this window. Figure 4 shows $\xi_{p}$ as a function of $p$, exhibiting a clear departure from the linear behavior (dashed line) and thus intermittent statistics.

Moreover, we have verified the exact result derived by two of us (G.D. and G.K.) for thin elastic plates that is valid for both weak and strong wave turbulence [12]. This result is equivalent to the only exact result of hydrodynamic turbulence, known as the 4/5 Kolmogorov law of turbulence. It relates increments of the field with the energy flux and the scales. In the case of a thin elastic plate, a similar relation was found for a different structure function, called $Z(\ell)$, that depends on the first-order variation of the fields $\chi, \zeta$, and $\dot{\zeta}$ (see Appendix B). In analogy with the 4/5 Kolmogorov law, this relation was called the 1 law and reads

$$
Z(\ell)=-P \ell
$$

The structure function $Z(\ell)$, normalized by $P \ell$, is displayed in the (top) inset of Fig. 4 . An excellent agreement with the prediction (14) is observed over a decade in space with no adjustable parameter. Finally, we have also studied the vertical speed structure function $\left\langle[\dot{\zeta}(\boldsymbol{r}+\boldsymbol{\ell})-\dot{\zeta}(\boldsymbol{r})]^{3}\right\rangle$. Unlike hydrodynamic turbulence, it does not scale linearly with the distance $\ell$. This difference can be explained by the existence of the extra-length $\lambda$, suggesting that the exponent cannot be uniquely determined by dimensional analysis. In addition, this third-order correlation function is not as simply related to the energy transfer as in the case of hydrodynamics. This fact is due to the complexity of the nonlinear term, which is precisely taken into account in (14). 


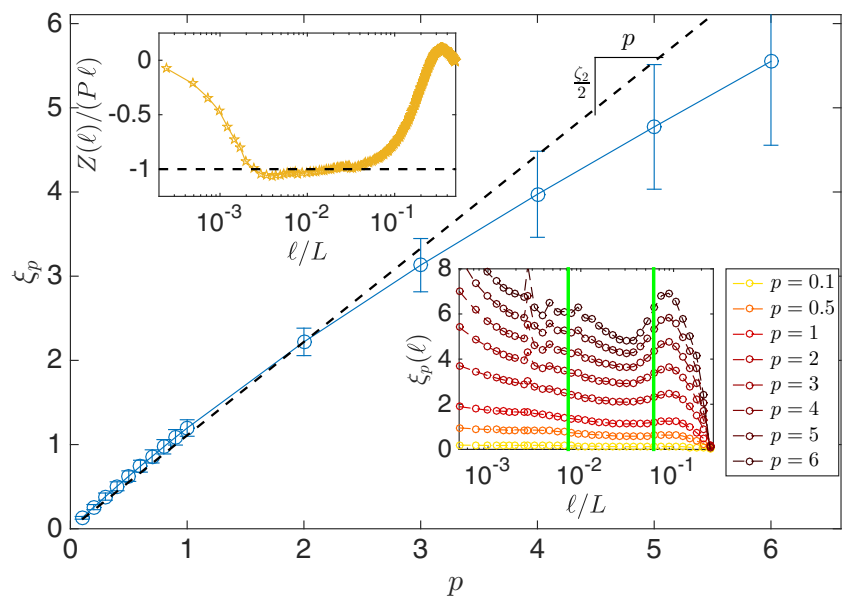

FIG. 4. Intermittency exponents $\xi_{p}$ as a function of $p$ clearly showing a departure from a linear law, indicating the existence of anomalous exponents and intermittency. The lower-right inset shows the local slopes of the structure functions as a function of $\ell$. The inertial range is delimited by the two vertical green lines. The upper-left panel shows evidence of the 1 law (14). The numerics were done under the same conditions as the previous figures.

\section{DISCUSSION}

Our results suggest that strong turbulence is a rather universal phenomenon, where a clear analogy can be established at least between ordinary fluids and crumpling vibrations of elastic plates in the zero-limit thickness. As presented, both exhibit an energy cascade with the well-known K41 spectrum, $E_{k} \sim P^{2 / 3} k^{-5 / 3}$. More importantly, a myriad of randomly interacting, highly nonlinear crumpling structures (folds, ridges, and D cones) at all relevant scales dominates the dynamics. They induce intermittency that we have quantitatively investigated by studying high-order correlation functions that confirm the appearance of an intermittent behavior. Nevertheless, the underlying plate dynamics differ notably from those of ordinary fluids: while in incompressible fluids the total energy consists purely of kinetic energy, in elastic plates the energy is composed of two separately (positive) energies: the kinetic and the stretching energy. We have observed in our numerics that the cascade of energy was in fact a cascade of the kinetic contribution. This is because the forcing is additive to the deformation acceleration (1), imposing a kinetic-energy flux [see Fig. 3(a)] resulting in a K41 cascade. On the other hand, the stretching energy flux vanishes [see Fig. 3(a)] and, consequently, Gibbs equipartition is observed for the stretching spectrum, as shown in the inset of Fig. 3(b). Naturally, it is expected that other forcing may display either a stretching energy cascade without any kinetic-energy cascade or perhaps two distinct cascades simultaneously, i.e., a kinetic-energy K41 cascade and a stretching one.

Finally, one may wonder whether a similar picture can be observed for other wave systems in the strong nonlinear limit. The first example that comes to mind is the dynamics of surface waves in the special limits of zero gravity and zero surface tension so that no linear waves exist. Interestingly, only the kinetic bulk energy contributes:

$$
H=\frac{\rho}{2} \int_{-\infty}^{\zeta(x, y, t)} d z \iint|\boldsymbol{v}|^{2} d x d y
$$

so that, by pure dimensional analysis, the K41 spectrum (9) should be expected.

Although the mathematics looks similar, the physical situation is more subtle. First, the interface motion is typically considered as an irrotational and incompressible flow in three dimensions, in which case the underlying equations of such free boundary problem are tractable theoretically. 
Second, the kinetic energy considered above is the one of the total fluid volume that cannot be linked directly to a "surface kinetic energy" or to any spectrum.

Naturally, the nonvortical character of strong wave turbulence under such approximation is questionable with regards to the usual fully developed turbulent behavior expected here. It raises different questions for this fluid analogy that might be worth investigating in future works: can a K41 spectrum arise in this zero gravity and surface tension limit? How can such K41 spectrum be compatible with the three-dimensional flow? Finally, such physical situation could be encountered experimentally by considering superfluid helium in the $0 \mathrm{~K}$ temperature limit and in a low- (ideally zero-)gravity context.

\section{ACKNOWLEDGMENTS}

G.D., G.K., and S.R. are thankful for FONDECYT Grant No. 1181382 and the Chilean-French scientific exchange program ECOS-Sud/CONICYT Grant No. C14E04. S.R. thanks the Gaspard Monge Visiting Professor Program of École Polytechnique (France).

\section{APPENDIX A: THE ENERGY SPECTRA AND ENERGY FLUXES}

The energies in Eqs. (3) and (4) may be seen as a quadratic contribution of $\dot{\zeta}$ and

$$
\gamma(\boldsymbol{r})=-\Delta^{-1}\left(\zeta_{x x} \zeta_{y y}-\zeta_{x y}^{2}\right)
$$

The final quadratic contributions read

$$
\begin{aligned}
\mathcal{E}_{\text {kin }} & =\frac{1}{2} \int\left|\dot{\zeta}_{\boldsymbol{k}}\right|^{2} d \boldsymbol{k} \\
\mathcal{E}_{\text {stret }} & =\frac{E}{2 \rho} \int\left|\gamma_{\boldsymbol{k}}\right|^{2} d \boldsymbol{k}
\end{aligned}
$$

Thus, we define the kinetic-energy and the stretching spectra by averaging over the angular variables in the wave-number space; hence,

$$
\begin{aligned}
E_{\text {kin }}(k) & =\pi k\left\langle\left|\dot{\zeta}_{k}\right|^{2}\right\rangle, \\
E_{\text {stret }}(k) & =\pi \frac{E}{\rho} k\left\langle\left|\gamma_{k}\right|^{2}\right\rangle,
\end{aligned}
$$

where we have defined $\langle\cdot\rangle=\frac{1}{2 \pi} \int \ldots d \varphi_{k}$ by the angular average.

Because the energy is quadratic in $\dot{\zeta}$ and $\gamma$, the energy flux can be straightforwardly defined as in hydrodynamic turbulence. By making a scale-by-scale energy budget, the energy fluxes are

$$
\begin{gathered}
P_{\text {kin }}(k)=-\left.\int_{0}^{k} \frac{\partial E_{\mathrm{kin}}(p)}{\partial t}\right|_{0} d p=-\int_{0}^{k}\left\langle\dot{\zeta}_{p}\{\zeta, \chi\}_{-p}\right\rangle d p, \\
P_{\text {stret }}(k)=-\left.\int_{0}^{k} \frac{\partial E_{\text {stret }}(p)}{\partial t}\right|_{0} d p=\int_{0}^{k}\left\langle\chi_{p}\{\zeta, \dot{\zeta}\}_{-p}\right\rangle d p,
\end{gathered}
$$

where the subscript 0 stands for the free of forcing and dissipation time variations of the fields through (1) with $\mathcal{F}=\mathcal{D}=0$. For simplicity, we define $\{f, g\} \equiv f_{x x} g_{y y}+f_{y y} g_{x x}-2 f_{x y} g_{x y}$. We have also used the fact that the angular averages satisfy $\left\langle\left(\Delta^{-1} f\right)_{p}\left(\Delta^{-1} g\right)_{-p}\right\rangle=\left\langle\left(\Delta^{-2} f\right)_{p} g_{-p}\right\rangle$. The totalenergy flux results from the addition of these previous expressions. 


\section{APPENDIX B: LAW FOR THIN ELASTIC PLATE TURBULENCE}

In Ref. [12], an exact result for turbulence of thin elastic plates was found. It provides a KármánHowarth-Monin-type relationship for the energy flux,

$$
\frac{1}{2} \nabla_{\ell} \cdot\left\langle\boldsymbol{J}_{[\delta \chi, \delta \zeta]} \delta \dot{\zeta}\right\rangle=-P
$$

where $\delta$ stands for the first difference of the field, e.g., $\delta \zeta=\zeta(\boldsymbol{x}+\boldsymbol{\ell})-\zeta(\boldsymbol{x})$. The vector $\boldsymbol{J}$ is defined by

$$
\boldsymbol{J}_{[f, h]}=f_{y} h_{y x}-f_{x} h_{y y}, f_{x} h_{x y}-f_{y} h_{x x} .
$$

Under the assumption of isotropy, (B1) implies the 1 law (14) for the structure function,

$$
Z(\ell) \equiv\left\langle\boldsymbol{J}_{[\delta \chi, \delta \zeta]} \delta \dot{\zeta}\right\rangle \cdot \hat{\ell}=-P \ell,
$$

where $\hat{\ell}$ is the unit vector along $\ell$.

[1] O. Reynolds, An experimental investigation of the circumstances which determine whether the motion of water shall be direct or sinuous, and of the law of resistance in parallel channels, Phil. Trans. R. Soc. London 174, 935 (1883).

[2] A. N. Kolmogorov, Dissipation of energy in the locally isotropic turbulence, Dokl. Akad. Nauk SSSR 32, 19 (1941) [Reprinted in Proc. Royal Soc.: Math. Phys. Sci. 434, 15 (1991)].

[3] J. I. Cardesa, A. Vela-Martín, and J. Jimenez, The turbulent cascade in five dimensions, Science 357, 782 (2017).

[4] D. Castelvecchi, Mysteries of turbulence unravelled, Nature (London) 548, 382 (2017).

[5] D. P. Ruelle, Hydrodynamic turbulence as a problem in nonequilibrium statistical mechanics, Proc. Natl. Acad. Sci. USA 109, 20344 (2012).

[6] T. von Kàrman and L. Howarth, On the statistical theory of isotropic turbulence, Proc. R. Soc. London 164, 192 (1938).

[7] U. Frisch, Turbulence: The Legacy of A.N. Kolmogorov (Cambridge University Press, Cambridge, 1995).

[8] M. K. Verma, Statistical theory of magnetohydrodynamic turbulence: Recent results, Phys. Rep. 401, 229 (2004).

[9] A. Groisman and V. Steinberg, Elastic turbulence in a polymer solution flow, Nature (London) 405, 53 (2000).

[10] A. Föppl, Vorlesungen über Technische Mechanik, Bd. 5 (Druck und Verlag von B.G. Teubner, Leipzig, 1907), p. 132.

[11] T. von Kàrman, Festigkeitsprobleme im maschinenbau, Ency. d. Math. Wiss., Bd. IV, Art. 27. Sec. 8 (Druck und Verlag von B.G. Teubner, Leipzig, 1910), p. 348.

[12] G. Düring and G. Krstulovic, Exact result in strong wave turbulence of thin elastic plates, Phys. Rev. E 97, 020201(R) (2018).

[13] G. Düring, C. Josserand, and S. Rica, Weak Turbulence for a Vibrating Plate: Can One Hear a Kolmogorov Spectrum? Phys. Rev. Lett. 97, 025503 (2006).

[14] A. Boudaoud, O. Cadot, B. Odille, and C. Touzé, Observation of Wave Turbulence in Vibrating Plates, Phys. Rev. Lett. 100, 234504 (2008).

[15] N. Mordant, Are There Waves in Elastic Wave Turbulence? Phys. Rev. Lett. 100, 234505 (2008).

[16] K. Hasselmann, On the nonlinear energy transfer in a gravity-wave spectrum. Part 1. General theory, J. Fluid Mech. 12, 481 (1962).

[17] K. Hasselmann, On the nonlinear energy transfer in a gravity-wave spectrum. Part 2. Conservation theorems; wave-particle analogy; irreversibility, J. Fluid Mech. 15, 273 (1963).

[18] K. Hasselmann, On the nonlinear energy transfer in a gravity-wave spectrum. Part 3. Evaluation of the energy flux and swell-sea interaction for a Neumann spectrum, J. Fluid Mech. 15, 385 (1963). 
[19] D. J. Benney and P. G. Saffman, Nonlinear interactions of random waves in a dispersive medium, Proc. R. Soc. London, Ser. A 289, 301 (1966).

[20] V. E. Zakharov, V. S. L'vov, and G. Falkovich, Kolmogorov Spectra of Turbulence I (Springer, Berlin, 1992).

[21] V. E. Zakharov and N. N. Filonenko, Energy spectrum for stochastic oscillations of the surface of a fluid, Dokl. Akad. Nauk. SSSR 170, 1292 (1966) [Sov. Phys. Dokl. 11, 881 (1967)].

[22] V. E. Zakharov, Weak turbulence spectrum in a plasma without a magnetic field, Zh. Eksper. Teoret. Fiz. 51, 688 (1966) [Sov. Phys. JETP 24, 455 (1967)].

[23] V. E. Zakharov and N. N. Filonenko, Weak turbulence of capillary waves, Zh. Prikl. Mekh. I Tekhn. Fiz. 8, 62 (1967) [J. Appl. Mech. Tech. Phys. 8, 37 (1967)].

[24] D. J. Benney and A. C. Newell, Sequential time closures for interacting random waves, J. Math. Phys. 46, 363 (1967).

[25] A. C. Newell, The closure problem in a system of random gravity waves, Rev. Geophys. 6, 1 (1968).

[26] D. J. Benney and A. C. Newell, Random wave closures, Stud. Appl. Math. 48, 29 (1969).

[27] S. Dyachenko, A. C. Newell, A. Pushkarev, and V. E. Zakharov, Optical turbulence: Weak turbulence, condensates and collapsing filaments in the nonlinear Schrödinger equation, Physica D 57, 96 (1992).

[28] S. Galtier and S. V. Nazarenko, Turbulence of Weak Gravitational Waves in the Early Universe, Phys. Rev. Lett. 119, 221101 (2017).

[29] L. D. Landau and E. M. Lifshitz, Theory of Elasticity (Pergamon, New York, 1959).

[30] G. Düring, C. Josserand, and S. Rica, Wave turbulence theory of elastic plates, Physica D 347, 42 (2017).

[31] T. A. Witten and H. Li, Asymptotic shape of a Fullerene ball, Europhys. Lett. 23, 51 (1993).

[32] M. Ben Amar and Y. Pomeau, Crumpling paper, Proc. R. Soc. A 453, 729 (1997).

[33] E. Cerda and L. Mahadevan, Conical Surfaces and Crescent Singularities in Crumpled Sheets, Phys. Rev. Lett. 80, 2358 (1998).

[34] See Supplemental Material at http://link.aps.org/supplemental/10.1103/PhysRevFluids.4.064804 for movies of these defect-induced dynamics.

[35] M. K. Verma and D. Donzis, Energy transfer and bottleneck effect in turbulence, J. Phys. A 40, 4401 (2007).

[36] A. C. Newell, S. Nazarenko, and L. Biven, Wave turbulence and intermittency, Physica D 152-153, 520 (2001).

[37] E. Falcon, S. Fauve, and C. Laroche, Observation of Intermittency in Wave Turbulence, Phys. Rev. Lett. 98, 154501 (2007).

[38] N. Mordant and B. Miquel, Intermittency and Emergence of Coherent Structures in Wave Turbulence of a Vibrating Plate, Phys. Rev. E 96, 042204 (2017).

[39] N. Yokoyama and M. Takaoka, Weak and strong wave turbulence spectra for elastic thin plate, Phys. Rev. Lett. 110, 105501 (2013).

[40] S. Chibbaro and C. Josserand, Elastic wave turbulence and intermittency, Phys. Rev. E 94, 011101(R) (2016). 